\title{
Matching top-bottom processes in charged Higgs production
}

\section{Johan Alwall*}

Université catholique de Louvain (Belgium)

E-mail: alwall@fyma.ucl.ac.be

Top is a very important probe to test theories for the electroweak symmetry breaking such as two-Higgs doublet models. One process of great interest in theories with extended Higgs sectors is the associated production of top, bottom and charged Higgs, described by $g b \rightarrow H^{-} t$ (where the extra $\bar{b}$ is described by parton showering) and $g g \rightarrow H^{-} t \bar{b}$. To get a reliable description of this production when the extra b-quark is observed, these processes must be matched to remove double-counting, a problem similar to that in the single top processes $p p \rightarrow t b W^{ \pm}$and $u b \rightarrow d t$. In this talk, we present our method for doing this matching at a fully differential level, and its implementation in the event generator Pythia. The implementation files can be downloaded from http://www.isv.uu.se/thep/MC/matchig/ .

International Workshop on Top Quark Physics

January 12-15, 2006

Coimbra, Portugal

* Speaker. 


\section{Introduction}

The existence of a charged Higgs boson is a common feature of many extensions of the Standard Model of particle physics, most notably supersymmetric extensions such as the MSSM, where (at least) two Higgs doublets are necessary to allow mass generation for both up- and down-type fields, and for anomaly cancellation. After electroweak symmetry breaking, the two Higgs doublets give rise to five physical scalar fields, $h, H, A, H^{+}$and $H^{-}$, where the $A$ is CP-odd assuming $\mathrm{CP}$ conservation (in the $\mathrm{CP}$-violating case, all three neutral scalars mix).

In the MSSM, two parameters are sufficient to determine the masses and interactions of these fields: $\tan \beta=\frac{v_{1}}{v_{2}}$, the ratio of the vacuum expectation values for the two Higgs doublets, and one of the masses, usually chosen as the pseudoscalar mass $M_{A}$. In a general two Higgs doublet model (2HDM) there are up to eleven parameters.

Needless to say, the discovery of a charged scalar particle would be a clear signal of physics beyond the Standard Model. In order to search for such a particle, we need an accurate description of the production mechanisms and phase-space distributions. Using Monte Carlo event generators, the production of charged Higgs can then be simulated, allowing for optimization of search strategies. Due to the large top Yukawa coupling, processes involving top quarks tend to dominate the production of charged Higgs bosons. Therefore a precise description of such processes are of vital importance in the future search for charged Higgs bosons.

In Monte Carlo generators such as Pythia[1] and HeRwig[2], the production channels used to simulate single charged Higgs boson production are $g b \rightarrow H^{-} t$ and $g g \rightarrow H^{-} t \bar{b}$ and their charge conjugates. (The additional process $q \bar{q} \rightarrow H^{ \pm} t b$ gives a large contribution in a $p \bar{p}$-collider such as Tevatron, but only a very small contribution at the high energies of the LHC. It is not affected by the matching problems discussed here and will therefore be disregarded in the following.) The $g g \rightarrow H^{ \pm} t b$ process gives a better description of the part of phase-space where the outgoing $b$ quark has a large transverse momentum, while the $g b \rightarrow H^{ \pm} t$ process resums potentially large logarithms $\left(\alpha_{s} \log \left(\mu_{F} / m_{b}\right)\right)^{n}$ and hence give a better description of the total cross-section and the (dominating) part of the phase space where the outgoing $b$-quark is collinear with the beam. However, in this region, where the outgoing $b$-quark has small transverse momentum, the two processes overlap, leading to double-counting in this region of phase-space. Together with Johan Rathsman at Uppsala University, we have developed a method to remove this double-counting by generating events from a distribution corresponding to the double-counted part of phase space, and subtracting these events from the sum. This is especially important in the poorly understood region of parameter space where the charged Higgs mass is similar to the top mass, and the two processes are of similar order of magnitude. Our work is presented in [3], where also more references are found. The algorithm is implemented as an external process to the PYTHIA event generator, and the files for this can be found at http://www.isv.uu.se/thep/MC/matchig/together with a manual.

\section{The twin-processes and their double-counting}

As discussed in the introduction, the two tree-level processes used in standard Monte Carlo 


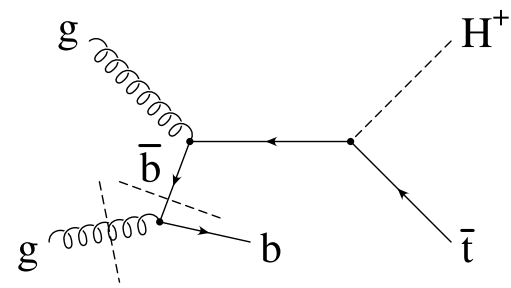

Figure 1: Illustration of the relation between the $g b \rightarrow H^{ \pm} t$ and $g g \rightarrow H^{ \pm} t b$ processes. If the factorization between the parton densities and the hard scattering is done at the gluon line we get the $g g \rightarrow H^{+} \bar{t} b$ process, while if instead this factorization is done at the $\bar{b}$ line, we get the $g \bar{b} \rightarrow H^{+} \bar{t}$ process. They can therefore be viewed as the same process in two different approximations.

simulation of single production of charged Higgs at hadron colliders are

$$
\begin{aligned}
& g b(\bar{b}) \rightarrow t H^{-}\left(\bar{t} H^{+}\right) \\
& g g \rightarrow t \bar{b} H^{-}\left(\bar{t} b H^{+}\right)
\end{aligned}
$$

The first one (2.1), which will be denoted the $2 \rightarrow 2$ process, includes the $b$-quark density, $b\left(\mu_{F}^{2}\right) \sim$ $\sum\left(\alpha_{s} \log \left(\mu_{F} / m_{b}\right)\right)^{n}$, which comes from the logarithmic DGLAP resummation of gluon splittings to $b \bar{b}$ pairs. This means that the $b$-quark going into the process is accompanied by a $\bar{b}$ (or vice versa) which is not explicitly shown in the equation. Due to the approximation made in the DGLAP expansion, this accompanying $b$-quark is nearly collinear with the beam. The second production process, (2.2), which will be denoted the $2 \rightarrow 3$ process, gives the correct treatment of the kinematics of the accompanying $b$-quark to order $\alpha_{s}^{2}$. The relation between the two processes is illustrated in fig. 1. Since the processes have the same initial and final states, they can be viewed as the same process in two different approximations, hence the term "twin-processes".

As suggested by fig. 1, there is an overlap between the two processes: When the transverse momentum of the outgoing $b$-quark in the $2 \rightarrow 3$ process is small, there is no distinction between the full $2 \rightarrow 3$ matrix element and a gluon splitting to $b \bar{b}$ convoluted with the $g b \rightarrow H^{ \pm} t$ matrix element. Therefore there is a double-counting between the processes, which can be expressed as [4]

$$
\sigma_{\mathrm{DC}}=\int d x_{1} d x_{2}\left[g\left(x_{1}, \mu_{F}\right) b^{\prime}\left(x_{2}, \mu_{F}\right) \frac{d \hat{\sigma}_{2 \rightarrow 2}}{d x_{1} d x_{2}}\left(x_{1}, x_{2}\right)+x_{1} \leftrightarrow x_{2}\right]
$$

where

$$
\begin{aligned}
b^{\prime}\left(x, \mu_{F}^{2}\right) & =\frac{\alpha_{s}\left(\mu_{R}^{2}\right)}{2 \pi} \int \frac{d Q^{2}}{Q^{2}+m_{b}^{2}} \int \frac{d z}{z} P_{g \rightarrow q \bar{q}}(z) g\left(\frac{x}{z}, Q^{2}\right) \\
& \approx \frac{\alpha_{s}\left(\mu_{R}^{2}\right)}{2 \pi} \log \frac{\mu_{F}^{2}}{m_{b}^{2}} \int \frac{d z}{z} P_{g \rightarrow q \bar{q}}(z) g\left(\frac{x}{z}, \mu_{F}^{2}\right)
\end{aligned}
$$

This is just the leading logarithmic contribution to the $b$-quark density included in the $2 \rightarrow 2$ process. Here $P_{g \rightarrow q \bar{q}}(z)=\frac{1}{2}\left[z^{2}+(1-z)^{2}\right]$ is the splitting function for $g$ going to $q \bar{q}, \mu_{F}$ is the factorization scale, $\mu_{R}$ is the renormalization scale used in evaluating $\alpha_{s}$, and $Q^{2}=-k^{2}$, the 4momentum of the incoming $b$-quark squared. We need to take care to include kinematic constraints 


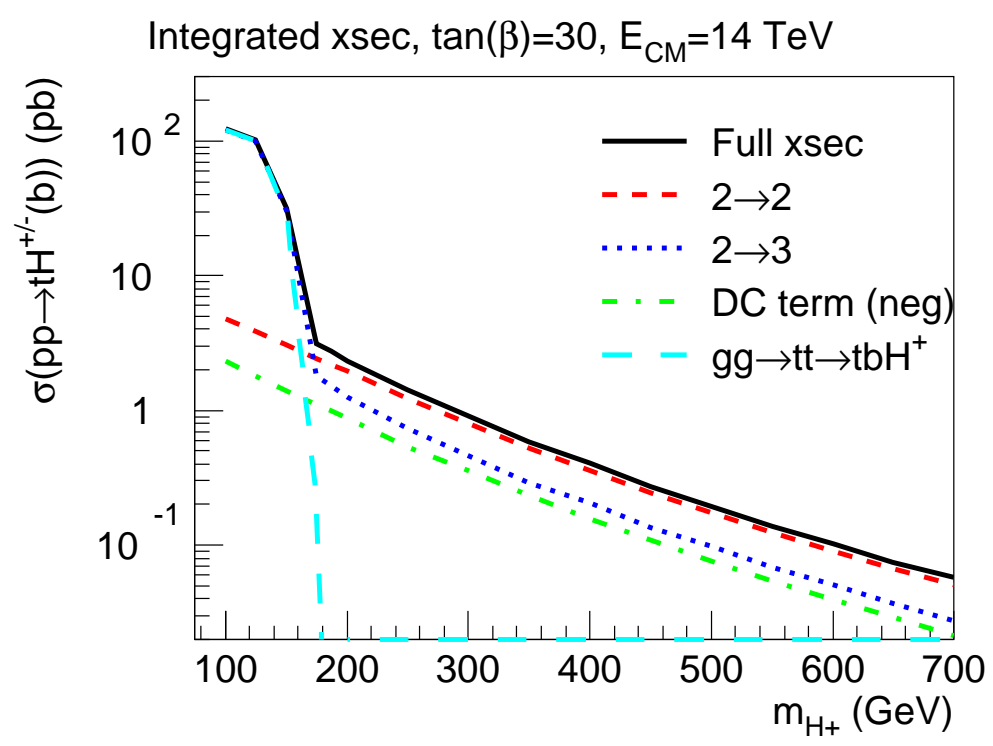

Figure 2: Integrated cross-section components (leading order process, $2 \rightarrow 3$ process and double-counting term) and matched total as a function of the $H^{ \pm}$mass at LHC, with $\tan \beta=30$ and $\mu_{F}=\left(m_{t}+m_{H^{ \pm}}\right) / 4$. Note that the double-counting term contribution (DC) is subtracted from the sum. At $m_{H^{ \pm}}<m_{t}$ the $2 \rightarrow 3$ process can be approximated by $g g \rightarrow t \bar{t} \rightarrow t b H^{ \pm}$.

due to the finite center-of-mass energy in our calculation of the integration limits, since such constraints are implicitly included in the $2 \rightarrow 3$ matrix element. This is done in detail in [3].

The matched integrated cross-section is then given by

$$
\sigma=\sigma_{2 \rightarrow 2}+\sigma_{2 \rightarrow 3}-\sigma_{\mathrm{DC}}
$$

The matched integrated cross-section and its components are shown as a function of the charged Higgs boson mass in fig. 2, For charged Higgs masses below the top mass the crosssection is dominated by top pair production with subsequent decay of one of the top quarks to $H^{ \pm}$b, i.e. $g g \rightarrow t \bar{t} \rightarrow t b H^{ \pm}$(for a comparison between this process and the complete $2 \rightarrow 3$ process, see [5]). For large charged Higgs masses $m_{H^{ \pm}}>m_{t}$, the $2 \rightarrow 2$ process dominates. Our matching procedure works for all charged Higgs masses, but is of greatest interest for $m_{H^{ \pm}} \gtrsim m_{t}$ and especially in the region $m_{H^{ \pm}} \sim m_{t}$, where the two processes are of similar order of magnitude. In the following we will use $m_{H^{ \pm}}=250 \mathrm{GeV}$ and $\tan \beta=30$ as a case study, where the features of the matching are clearly visible.

\section{Matching the differential cross-sections}

For the differential cross-section, we need to make sure some basic requirements are fulfilled:

1. The integrated cross-section should equal the correct one given by eq. (2.6).

2. All differential cross-sections should be smooth after matching. 

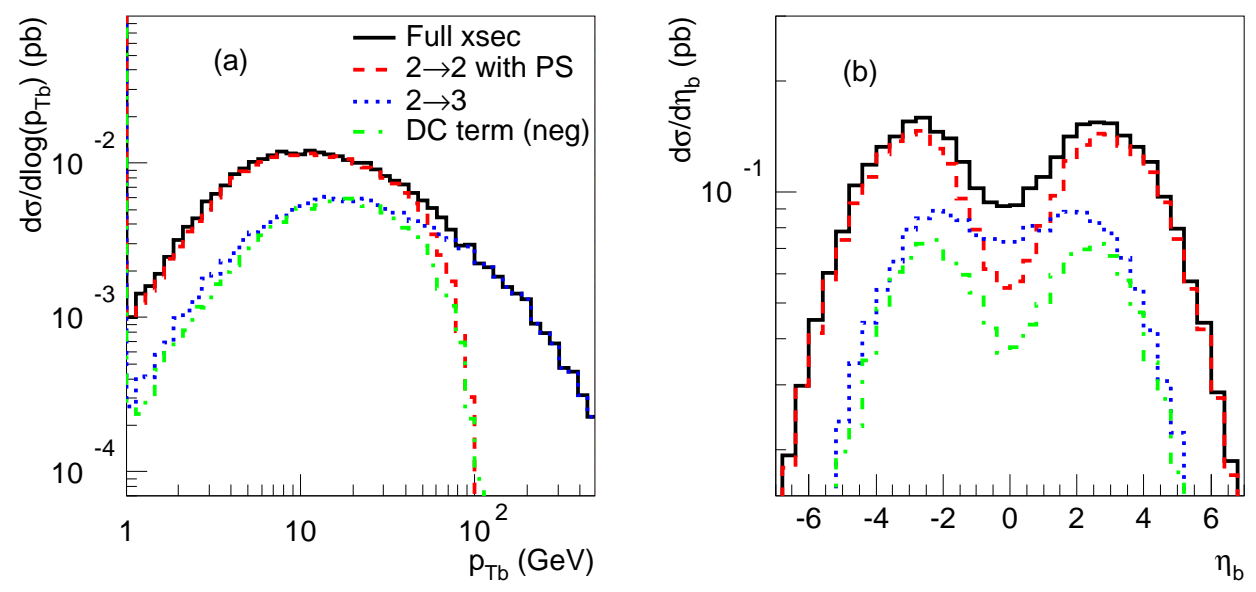

Figure 3: Differential cross-sections in (a) $p_{T, b}$, (b) $\eta_{b}$ for the cross-section components and the resulting matched cross-section with $\tan \beta=30, m_{H^{ \pm}}=250 \mathrm{GeV}$ and $\mu_{F}=\left(m_{t}+m_{H^{ \pm}}\right) / 4$. Note that the doublecounting term contribution (DC) is subtracted from the sum.

3. The matched $p_{T}$-distribution for the outgoing $b$-quark should be given by the $2 \rightarrow 2$ process for small transverse momenta, and by the $2 \rightarrow 3$ process for large transverse momenta, with a smooth interpolation between those regions.

Our solution to the matching problem is simple: We view the double-counting term (given by eq. (2.3) ) as a probability distribution in kinematic variables and pick events from this distribution. This contribution is then subtracted (i.e. added with negative weight) from the sum of the two processes (2.1) and (2.2) in the final data analysis, i.e. the histograms.

One might worry that this procedure could give a negative number of events in some phasespace region. However, the leading-logarithmic part of the $b$ density used in the double-counting term is always smaller then the full $b$ density used in the $2 \rightarrow 2$ process, ensuring that if only a sufficiently large number of events is generated, the sum of the events from the $2 \rightarrow 2$ and the $2 \rightarrow 3$ process will always be larger than the number of events from the double-counting term.

Some resulting differential cross-sections from our matching are shown in fig. 3, Looking at fig. 3a we see that the matched differential cross-section in $p_{T}$ for the outgoing $b$-quark looks exactly as expected (and wanted): for small $p_{T}$ it follows the $2 \rightarrow 2$ process distribution, while for large $p_{T}$ it follows the $2 \rightarrow 3$ process. However, there is a rather large intermediate region, from about $30 \mathrm{GeV}$ to about $100 \mathrm{GeV}$, where the matching procedure is necessary to get the correct differential cross-section. For comparison one can note that at LHC, the region where $b$-quarks can be tagged is $p_{T, b} \gtrsim 20 \mathrm{GeV}$. In fig. 3b we see large differences in the rapidity distribution for the outgoing $b$-quark between the matched cross section and each of the two contributing processes, up to a factor $\simeq 2$ in the experimentally interesting region $\left|\eta_{b}\right|<2.5$, indicating that matching is really necessary to get a correct description of the $b$-quark kinematics.

Even if the outgoing $b$-quark is not observed, there are still some differences between the matched cross-section and the $2 \rightarrow 2$ process, which is usually used alone in this case. In the 

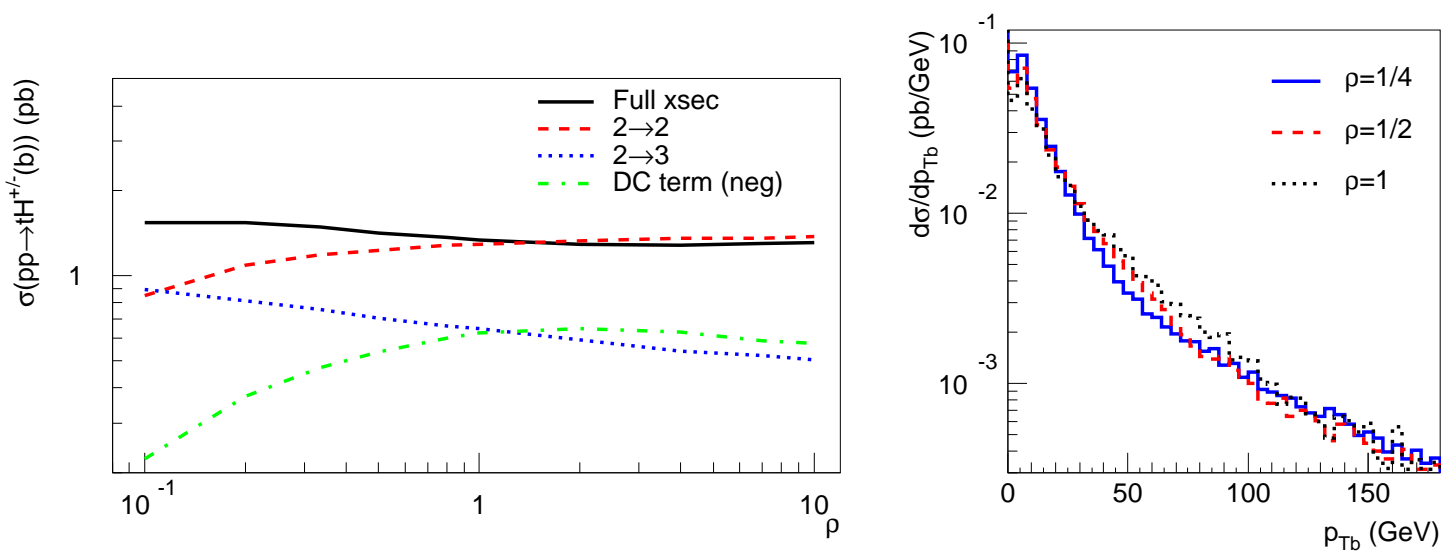

Figure 4: Left: Integrated cross-sections at $m_{H^{ \pm}}=250 \mathrm{GeV}$ and $\tan \beta=30$ as a function of the factorization scale parametrized by $\rho=2 \mu_{F} /\left(m_{t}+m_{H^{ \pm}}\right)$. Note that for $\rho \gtrsim 1$ the double-counting term exceeds the $2 \rightarrow 3$ term. Also note that the factorization scale dependence is smaller for the matched total cross-section than for any one of the components. Right: Matched differential cross-section in $p_{T}$ of the outgoing $b$-quark for three different values of $\rho$.

transverse momentum distribution of the $b$ coming from the decay of the top quark and the $\tau$ from the decay of the charged Higgs boson, the differences are of similar size as those obtained by turning off parton showers in the $2 \rightarrow 2$ process, see [3].

\section{Factorization scale dependence}

Fig. 4a shows the integrated cross-section components, as well as the matched total, as a function of the factorization scale $\mu_{F}$ parametrized by $\rho=\mu_{F} / \bar{m}$, where $\bar{m}=\left(m_{H^{ \pm}}+m_{t}\right) / 2$ is the average of the charged Higgs and top masses. Here two things can be noted. The first is that the matched integrated cross-section shows a significantly smaller dependence on the factorization scale than any one of the component cross-sections, indicating that the matching also stabilizes the cross-section. This is not surprising, since the $2 \rightarrow 3$ process is a part of the next-to-leading order calculation of the $2 \rightarrow 2$ process. Hence including it in a correct way should reduce the factorization scale dependence, as is the case when calculating the full next-to-leading order expression. The other thing to be noted is that the double-counting term exceeds the $2 \rightarrow 3$ process term from $\rho \gtrsim 1$, i.e. $\mu_{F} \gtrsim\left(m_{H^{ \pm}}+m_{t}\right) / 2$. Since the double-counting term of eq. (2.3) is designed to describe the part of the $2 \rightarrow 3$ process which is already included in the $2 \rightarrow 2$ process through the resummation of logarithmic contributions to the $b$-quark density, the double-counting term should not exceed the $2 \rightarrow 3$ process contribution in any part of the phase-space. Therefore this indicates that the factorization scale has been chosen too large. In fig. $4 \mathrm{~b}$ we see that the factorization dependence for the differential cross-section is also quite small, at most 20\%. This number should be compared with the difference between the $2 \rightarrow 2$ and $2 \rightarrow 3$-process differential cross-sections, which amounts to a factor $\sim 2$ at $p_{T, b} \sim 20 \mathrm{GeV}$. 


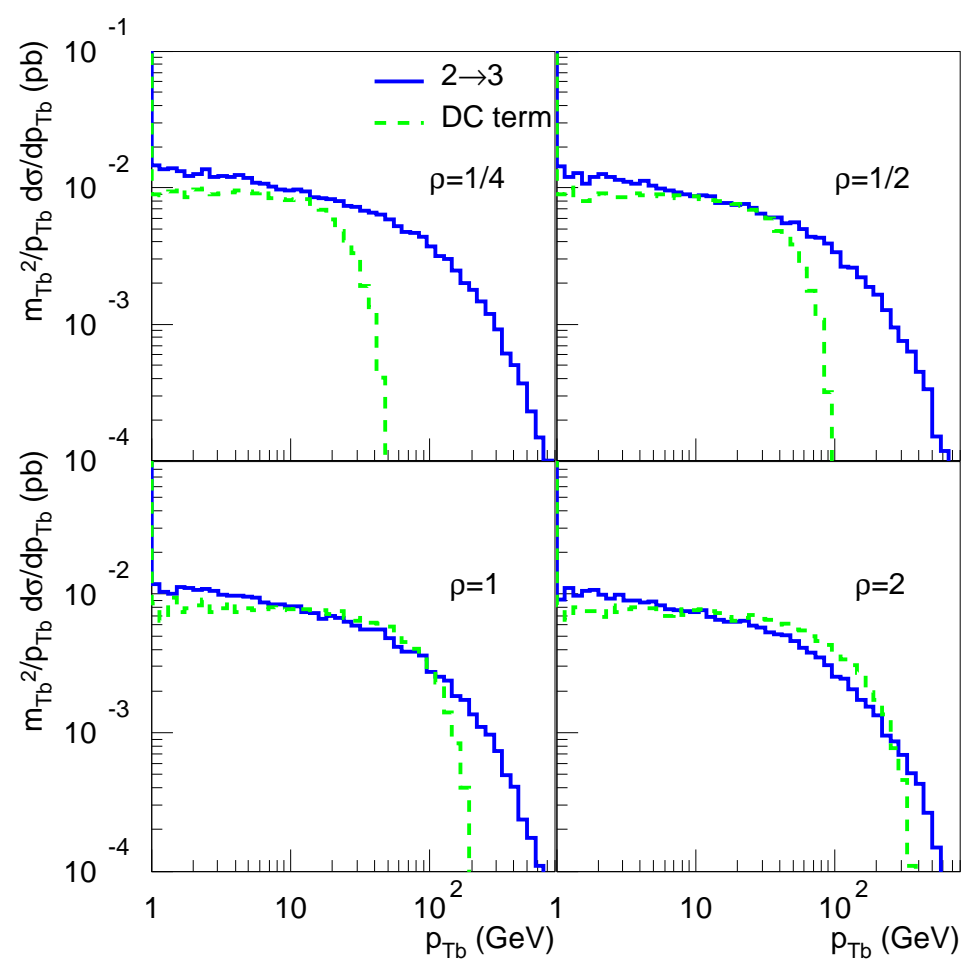

Figure 5: The differential cross-section $d \sigma / d p_{T, b}$ multiplied by $m_{T, b}^{2} / p_{T, b}$ for the $2 \rightarrow 3$ matrix element and the double-counting term for different factorization scales parameterized by $\rho=2 \mu_{F} /\left(m_{t}+m_{H^{ \pm}}\right)$. Note that the double-counting term overshoots the $2 \rightarrow 3$ term already for $\rho=1$.

The upper integration limit of the transverse momentum of the outgoing $b$-quark in the doublecounting term is determined by the factorization scale. Up to this limit the transverse momentumdistribution is almost flat, which means that the integrated value of the double-counting term is nearly proportional to the factorization scale (although for large scales the kinematic constraints modify this behaviour).

In view of the discussion above, the double-counting term should nowhere overshoot the $2 \rightarrow$ 3-term. In fig. 5 we see the distribution in $p_{T}$ of the outgoing $b$-quark for the $2 \rightarrow 3$ process (without parton showers) and the double-counting term at different factorization scales. Already for $\rho=1$, the double-counting term exceeds the $2 \rightarrow 3$ term around $p_{T, b} \approx 50 \mathrm{GeV}$. In fact, $\rho=0.5$, i.e. $\mu_{F}=\left(m_{H^{ \pm}}+m_{t}\right) / 4$, seems to be a limiting case where the double-counting distribution just touches the $2 \rightarrow 3$-distribution. Therefore we have chosen to use this (unconventionally small) value of the factorization scale in our plots in the previous sections. Similar results for the size of the factorization scale has been achieved in next-to-leading order calculations of the $2 \rightarrow 2$ process, see e.g. [6, 7].

\section{Application to other processes}

This approach for the subtraction of double-counting in $b$-quark initiated processes can be 
easily extended to other processes. These include, most naturally, charged flavour-changing heavy resonances coupling to top and bottom, such as a right-handed heavy $W^{ \pm}$, but also for example Standard Model single top production with $W^{ \pm}$-exchange in the t-channel (typically $u b \rightarrow t d$ ) and associated top and $W$ production, $g b \rightarrow t W^{ \pm}$.

It is also possible to extend the procedure to production of neutral resonances from $b$-quark fusion, such as $2 \mathrm{HDM}$ neutral Higgs boson production. There, the matching is more involved, since there are four different processes to be matched: $b \bar{b} \rightarrow H, b g \rightarrow H \bar{b}+$ c.c. and $g g \rightarrow H b \bar{b}$ (see [8] for a discussion of the double-counting structure in this process).

\section{Conclusions}

The discovery of a charged scalar particle would be a clear signal of physics beyond the Standard Model of particle physics. The detailed study of the properties of such a particle would give valuable insight into the nature of this new physics. But to be able to find the charged Higgs boson, we need to devise search strategies and reduce the Standard Model background. For this, an appropriate description of charged Higgs boson production, using Monte Carlo events generators, is necessary.

For single charged Higgs boson production, mainly two processes are used in Monte Carlos, the bottom-gluon fusion $g b \rightarrow H^{ \pm} t$ production channel and the gluon-gluon fusion $g g \rightarrow H^{ \pm} t b$ channel. These have different virtues: $g b \rightarrow H^{ \pm} t$ resums large logarithms describing the $b$-quark density, giving the major contribution to the total cross-section. It also gives the best description of the differential cross-section for small values of the transverse momentum of the accompanying $b$-quark. On the other hand, the $g g \rightarrow H^{ \pm} t b$ process gives a correct description to order $\alpha_{s}^{2}$ of the outgoing $b$-quark for large values of the transverse momentum. However, the two processes overlap in the small transverse momentum region, so in order to use them both we must compensate for this double-counting. This is especially important in the problematic parameter region where $m_{H^{ \pm}} \sim m_{t}$. There the two processes are of similar size, and therefore both need to be taken into account to get a reliable description of the charged Higgs boson production. A realistic study aiming to improve the discovery reach in this region of parameter space is underway, in collaboration with Atlas experimentalist Bjarte Mohn.

In this talk we have presented our algorithm for the matching of the two processes. This matching is done by summing the events from the two processes (as is usually done in Monte Carlo generators), and then subtract events generated from a double-counting distribution term from the sum. In this way we are able to combine the virtues of the two processes to get a reliable description of the differential cross-section.

This method also allows us to get a better understanding of what choice of factorization scale is appropriate, by comparing the transverse momentum distributions for the double-counting term with the distribution for the $2 \rightarrow 3$ process matrix element. Since the double-counting term should remove the part of the $2 \rightarrow 3$ distribution already contained in the $2 \rightarrow 2$ distribution, the doublecounting term should not overshoot the $2 \rightarrow 3$ term. The result is that the appropriate factorization scale turns out significantly smaller than the conventionally used value, in agreement with next-toleading order studies. 


\section{Acknowledgements:}

I would like to thank the organizers of TOP 2006 for giving me the opportunity to present this talk.

\section{References}

[1] T. Sjöstrand et al., Comput. Phys. Commun. 135 (2001) 238 [arXiv:hep-ph/0010017].

[2] G. Corcella et al., JHEP 0101 (2001) 010, [arXiv:hep-ph/0011363].

[3] J. Alwall and J. Rathsman, JHEP 0412 (2004) 050 [arXiv:hep-ph/0409094].

[4] F. Borzumati, J. L. Kneur and N. Polonsky, Phys. Rev. D 60 (1999) 115011 [arXiv:hep-ph/9905443].

[5] J. Alwall, C. Biscarat, S. Moretti, J. Rathsman and A. Sopczak, Eur. Phys. J. C 39S1 (2005) 37 [arXiv:hep-ph/0312301].

[6] T. Plehn, Phys. Rev. D 67 (2003) 014018 [arXiv:hep-ph/0206121].

[7] E. L. Berger, T. Han, J. Jiang and T. Plehn, Phys. Rev. D 71 (2005) 115012 [arXiv:hep-ph/0312286].

[8] D. Dicus, T. Stelzer, Z. Sullivan and S. Willenbrock, Phys. Rev. D 59 (1999) 094016 [arXiv:hep-ph/9811492]. 